\title{
Chemical Waves in Media with State-Dependent Anisotropy
}

\author{
N. Gottschalk, F. Mertens, M. Bär,* M. Eiswirth, and R. Imbihl* \\ Fritz-Haber-Institut der Max-Planck-Gesellschaft, Faradayweg 4-6, D-14195 Berlin (Dahlem), Germany
}

(Received 2 September 1994)

\begin{abstract}
In the reduction of $\mathrm{NO}$ with $\mathrm{H}_{2}$ on a $\mathrm{Rh}(110)$ surface rectangularly shaped target patterns and spirals with sharp corners have been observed. These patterns can be reproduced with a simple model assuming that the (anisotropic) diffusion is state dependent. Such a dependence is realized in the system $\mathrm{Rh}(110) / \mathrm{NO}+\mathrm{H}_{2}$ by the presence of different adsorbate-induced reconstructions with varying substrate geometries.
\end{abstract}

PACS numbers: 82.65.Jv, 05.70.Ln

The study of spatiotemporal self-organization on singlecrystal surfaces has revealed a large variety of chemical wave patterns in recent years [1-3]. A new aspect, which sets such systems apart from fluid-phase reactions, is the anisotropy of diffusion as a consequence of the rigid substrate geometry on which the adsorbed particles move. While simple anisotropy, i.e., an anisotropy which can be removed by rescaling the coordinates, only causes an elliptical distortion of the wave patterns, novel effects can occur if this rescaling is impossible [4,5]. Such a complex anisotropy occurs naturally in surface reactions where the anisotropy of surface diffusion depends on the state of the surface, i.e., on the adsorbate coverage and on the surface structure, giving rise to an anisotropy that varies along the profile of a chemical wave [6].

In this paper, we demonstrate that state-dependent diffusion decisively influences the shape of chemical waves, giving rise to a variety of patterns such as a transition from elliptical to rectangular waves. Experiments with the catalytic reduction of $\mathrm{NO}$ with $\mathrm{H}_{2}$ on a $\mathrm{Rh}(110)$ surface are presented, and it is shown that the observed effects can be reproduced with a standard model for reaction-diffusion systems if state-dependent anisotropy of diffusion is taken into account.

The catalytic reduction of $\mathrm{NO}$ with $\mathrm{H}_{2}$ to $\mathrm{N}_{2}$ and $\mathrm{H}_{2} \mathrm{O}$ proceeds on $\mathrm{Rh}(110)$ via the rapid dissociation of molecularly adsorbed NO into atomic oxygen and nitrogen [7]. We studied the reaction under isothermal low-pressure conditions in the $10^{-6}$ to $10^{-5}$ mbar range operating our ultrahigh vacuum system as a gradient-free flow reactor. The Rh(110) sample ( $8 \mathrm{~mm}$ diam) was polished with diamond paste (down to $0.025 \mu \mathrm{m}$ ) and cleaned in several sequences of exposure to oxygen, Ar + sputtering, and thermal annealing to $1370 \mathrm{~K}$. Spatially resolved measurements were conducted with photoemission electron microscopy (PEEM) [8]. This method images the locally varying work function, achieving a typical spatial resolution of $\approx 1 \mu \mathrm{m}$ in real time. Under reaction conditions at $T>500 \mathrm{~K}$, mainly atomic oxygen and nitrogen are present on the surface, and, since the highest work function increase is caused by atomic oxygen, we essentially image the oxygen concentration. Oxygen rich areas ap- pear dark and oxygen deficient areas bright in the PEEM images.

While the system at low temperature exhibits excitability, at high temperature $(T \approx 620 \mathrm{~K})$ we also observe bistability, but with both states undergoing dynamic changes via propagating reaction fronts [9]. As demonstrated by the PEEM images in Fig. 1(a), an elliptical oxygen front nucleates followed by a reducing front which is square shaped and removes the oxygen adlayer. Both the axes of the elliptical front as well as the sides of the rectangular front are oriented along the crystallographic directions of $\mathrm{Rh}(110)$. The front velocity in these experiments is of the order of a few $\mu \mathrm{m} / \mathrm{s}$.

In situ low energy electron diffraction measurements revealed the existence of $2 \times 1$ and $3 \times 1$ patterns indicative of nitrogen-induced reconstructions which can be associated with the bright areas in PEEM [10]. The darker areas in PEEM were found to be correlated with a $c(2 \times 6)$ pattern characteristic of adsorbed oxygen superimposed with a $c(2 \times 4)$ pattern which has been attributed to a mixed N,O adlayer [11,12]. All oxygeninduced reconstructions [including the mixed $c(2 \times 4)$ $\mathrm{N}, \mathrm{O}$ structure] are of the missing-row type with elongated troughs oriented along [1 $\overline{1} 0]$, while the nitrogen adstructures consist of $\mathrm{Rh}-\mathrm{N}$ chains in the [001] direction. The change in the row direction by $90^{\circ}$ as the reaction front progresses generates a strongly state-dependent anisotropy of surface diffusion which is the basis of the model discussed in the following.

Pattern formation on catalytic surfaces can typically be described by a two-component model with one fast activator variable $u$ representing an adsorbate coverage (changing rapidly due to adsorption and reaction processes) and a slow inhibitor variable $v$ representing the degree of surface reconstruction (acting as a negative feedback on the coverage) [13]. The following equations have been used:

$$
\begin{aligned}
& \partial_{t} u=\left(u-u^{3} / 3-v\right) / \varepsilon+\nabla[D(v) \nabla u], \\
& \partial_{t} v=u+b-a v .
\end{aligned}
$$

These equations are identical to the well known FitzHughNagumo model allowing oscillatory, excitable, or bistable local kinetics depending on the number of fixed points 

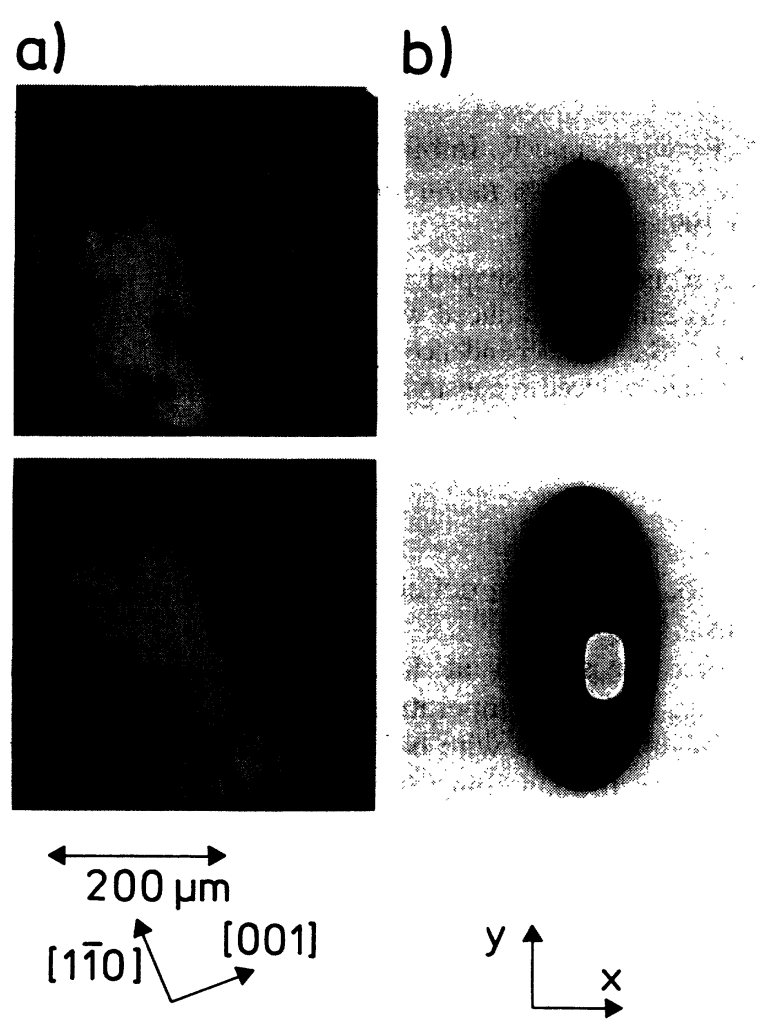

FIG. 1. Simultaneous observation of different front geometries during the $\mathrm{NO}+\mathrm{H}_{2}$ reaction on $\mathrm{Rh}(110)$. (a) Experiment. Two PEEM images recorded with a time interval of 19 s showing subsequent nucleation and growth of oxygen forming (black) and oxygen removing fronts (white). Experimental conditions $T=620 \mathrm{~K}, p_{\mathrm{NO}}=1.8 \times 10^{-6} \mathrm{mbar}$, and $p_{\mathrm{H}_{2}}=5.2 \times 10^{-6}$ mbar. (b) Simulation. Evolution of black and white fronts both from circular initial conditions (the white one was triggered in the first snapshot, 3 time units after the black one). The activator concentration is shown with black denoting higher values. $\left(a=2.1, b=-0.2, \varepsilon=0.025, D_{0}=\right.$ $0.25, D_{1}=1, D_{2}=3$, and $v_{\text {crit }}=0.4415$; explicit integration with $d t=0.001$, grid size $512 \times 512$ corresponding to $70 \times 70$ spatial units).

[14]. Here spatial coupling is introduced using a nonstandard diffusion term for the activator species. Since diffusion can change very rapidly with the degree of reconstruction $v$, we choose

$$
D_{x}=D_{0}, \quad D_{y}(v)= \begin{cases}D_{1}, & v \leq v_{\text {crit }}, \\ D_{2}, & v>v_{\text {crit }},\end{cases}
$$

i.e., the diffusion constant is only changed in the $y$ direction, but left unaltered in the $x$ direction. All calculations were also carried out with smoothed step functions (everywhere infinitely many times differentiable) which gave qualitatively the same results.

The remarkable effect seen in Fig. 1(a) that despite identical parameters different front geometries are observed can readily be explained with this simple model as demonstrated by Fig. 1(b). The concentration profile dis-

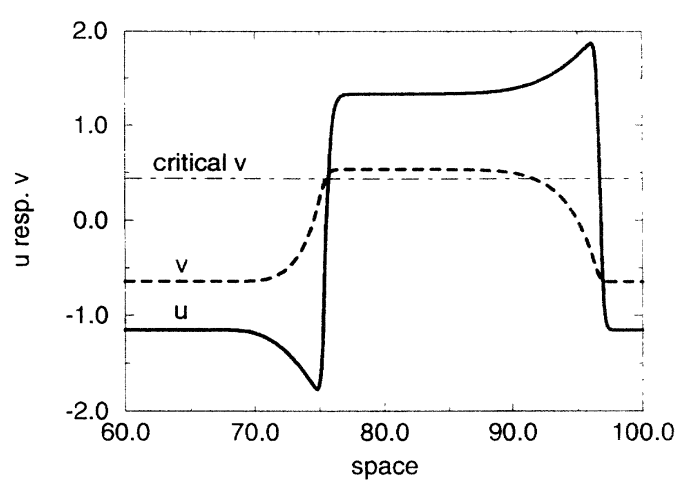

FIG. 2. Concentration profile to the simulation of Fig. 1(b) showing in the right part the transition from white to black ("black front") and in the left part the transition from black to white ("white front"). The dashed line indicates the critical value $v_{\text {crit }}$ of the inhibitor concentration at which the diffusional anisotropy of the activator switches [parameters as in Fig. 1(b)].

played in Fig. 2 yields the explanation for the different front geometries in the bistable case. Since the switching of the diffusional anisotropy can affect the shape of a wave front only if it happens in the immediate vicinity of the steepest concentration gradient of $u$, only the white front is affected, while the black one remains elliptical. In the excitable case, the leading front determines the shape of the pulses, and since, for the parameters chosen, the white front precedes the black front, distorted wave patterns can also be expected in this case.

Quite generally the deformation of wave patterns is governed by the angular dependence of the front velocity, $c(\alpha)$, with $\alpha$ denoting the angle with respect to a fixed surface direction. Caustics, i.e., patterns with sharp edges, show up when $c(\alpha)+\partial^{2} c / \partial \alpha^{2}$ becomes negative as demonstrated by Mikhailov [4]. While for simple anisotropic diffusion the relation $c \sim \sqrt{D}$ holds. this dependence is no longer valid for state-dependent anisotropy, and, moreover, no analytical expression for $c=f(\alpha)$ can be derived. This relationship can, however, be obtained by plane-wave simulations in different directions $\alpha$.

A surprising result of the simulations was that statedependent anisotropy can cause an effective slowing down of reaction fronts below the value given by the smallest diffusion coefficient, i.e., assuming $c_{f} \sim \sqrt{D_{\text {eff }}}$ one can have $D_{\text {eff }}<\min \left(D_{1}, D_{2}\right)$. Qualitatively this effect can be explained in the following way: If the activator diffusion in the region preceding the reaction front is fast, then this region acts as an effective sink for the activator concentration in the transition region where the switching to a lower diffusion constant occurs. It will therefore be more difficult for the activator to reach the excitation threshold, and this causes the slowing down of front propagation. An interesting consequence of this effect is that in media with state-dependent anisotropy the front may 


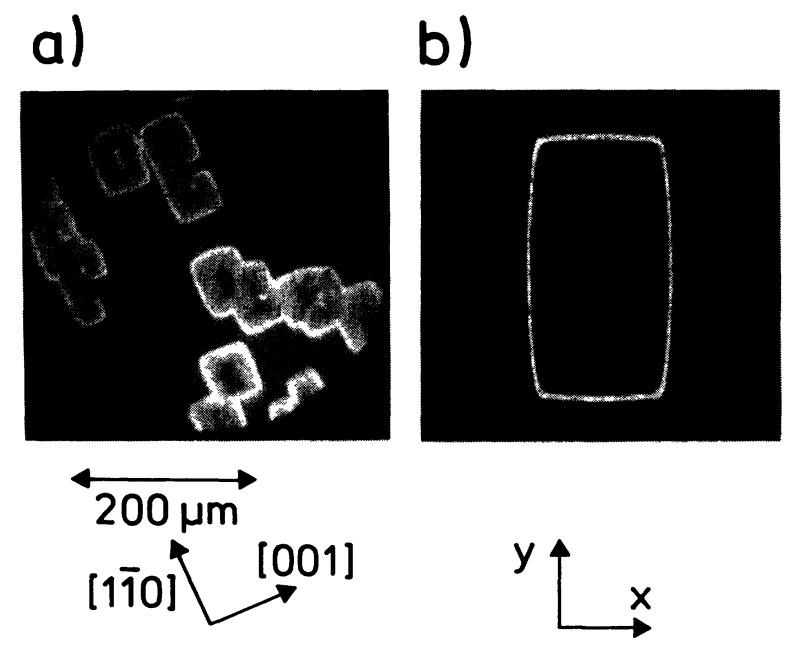

FIG. 3. Rectangularly shaped target pattern in the $\mathrm{NO}+$ $\mathrm{H}_{2}$ reaction on $\mathrm{Rh}(110)$ in the excitable regime. (a) Experiment. PEEM image recorded at $T=620 \mathrm{~K}$ with $p_{\mathrm{NO}}=$ $1.8 \times 10^{-6} \mathrm{mbar}$, and $p_{\mathrm{H}_{2}}=5 \times 10^{-6}$ mbar. (b) Simulation. Snapshot 7 time units after an initial condition of a white circle on the black rest state $[a=0.5, b=-1.04$, and other parameters as in Fig. 1(b).

actually propagate more slowly in the direction in which the diffusion is faster.

In the model the following typical scenario was found upon increasing the complex anisotropy (i.e., the difference between $D_{1}$ and $D_{2}$ ): At first the patterns were more and more deformed from the elliptical shape until sharp corners appeared (with diffusional rounding of the order of the front width). For even stronger anisotropy, propagation failure occurred in the $y$ direction, causing the two-dimensional patterns to break into wave segments propagating only in the $x$ direction. In the present system the above-mentioned transitions could also be introduced through variation of one of the parameters $a, b$, or $\varepsilon$ (for fixed $D_{1}$ and $D_{2}$ ), because a change in front or pulse shape alters the position in the transition region where the diffusional anisotropy switches.

This scenario could be observed in the experiment in the excitable regime. At $T<550 \mathrm{~K}$ one finds in PEEM elliptically shaped target patterns with the long and short axes oriented along the [110] and [001] directions, respectively [5]. Upon increasing the temperature the geometry of the wave patterns starts to deviate from ellipses until rectangularly shaped patterns are obtained. These patterns are displayed in Fig. 3 together with the corresponding computer simulations. By selecting suitable initial conditions, one may also observe rectangularly distorted spiral waves as demonstrated by the PEEM image in Fig. 4(a). Again, the simulation shown in Fig. 4(b) reproduces the experiment very well. The propagation failure described above was seen

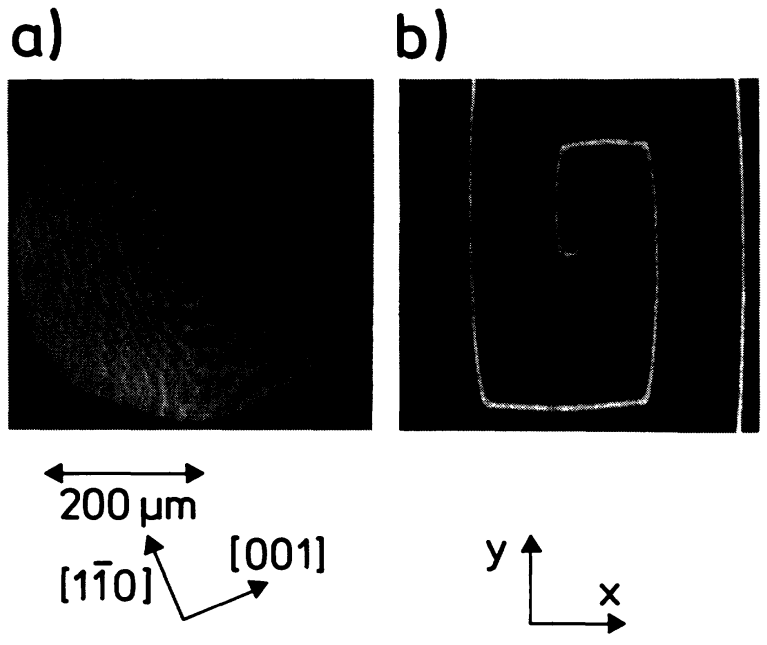

FIG. 4. Rectangularly distorted spiral wave in the $\mathrm{NO}+$ $\mathrm{H}_{2}$ reaction on $\mathrm{Rh}(110)$. (a) Experiment. PEEM image recorded at $T=560 \mathrm{~K}$ with $p_{\mathrm{NO}}=1.8 \times 10^{-6} \mathrm{mbar}$ and $p_{\mathrm{H}_{2}}=5.3 \times 10^{-6} \mathrm{mbar}$. The spiral rotates with a frequency of $4.3 \times 10^{-2} \mathrm{~s}^{-1}$. (b) Simulation. Spiral formed after 50 time units from cross-gradient initial conditions [parameters as in Fig. 3(b).

when $p_{\mathrm{H}_{2}}$ was decreased after initiating a target pattern [5]. The decrease caused those parts of the pattern oriented along [001] to dissolve leaving wave segments stretched along [110] which propagated in the [001] direction ["solitary waves" described earlier in the CO oxidation on $\operatorname{Pt}(110)$ [15]].

In summary, we have shown that state-dependent anisotropy of diffusion can generate novel and unexpected effects in chemical wave patterns. The effects shown here are not only of relevance to surface reactions, but should also play an important role in other anisotropic systems, e.g., chemical systems in the presence of external symmetry-breaking fields or biological systems.

Financial support from the Deutsche Forschungsgemeinschaft is gratefully acknowledged (F.M.). The authors are indebted to A. Mikhailov for valuable discussions.

*Present address: Department of Chemical Engineering, Princeton University, Princeton, NJ 09544-5263.

[1] G. Ertl, Science , 254, 1750 (1991).

[2] R. Imbihl, Prog. Surf. Sci. 44, 185 (1993).

[3] M. Eiswirth and G. Ertl, in Chemical Waves and Patterns, edited by R. Kapral and K. Showalter (Kluwer, Dordrecht, 1994).

[4] A. Mikhailov, Phys. Rev. E 49, 5875 (1994).

[5] F. Mertens and R. Imbihl, Nature (London) 370, 124 (1994).

[6] A. v. Oertzen, H. Rotermund, and S. Nettesheim, Surf. Sci. 311, 322 (1994). 
[7] M.F.H. van Tol, A. Gielbert, and B.E. Nieuwenhuys, Appl. Surf. Sci. 67, 179 (1993); Catal. Lett. 16, 297 (1992).

[8] W. Engel, M.E. Kordesch, H. H. Rotermund, S. Kubala, and A. von Oertzen, Ultramicroscopy 36, 148 (1991).

[9] S. Nettesheim, A. von Oertzen, H. H. Rotermund, and G. Ertl, J. Chem. Phys. 98, 9977 (1993).

[10] P. M. Murray, F. M. Leibsle, G. Thornton, M. Bowker, V. R. Dhanak, A. Baraldi, M. Kiskinova, and R. Rosei, Surf. Sci. 304, 48 (1994).
[11] F. M. Leibsle, P. W. Murray, S. M. Francis, G. Thornton, and M. Bowker, Nature (London) 363, 706 (1993).

[12] P. W. Murray, G. Thornton, M. Bowker, V. R. Dhanak, A. Baraldi, and M. Kiskinova, Phys. Rev. Lett. 71, 4369 (1993).

[13] M. Bär, N. Gottschalk, M. Eiswirth, and G. Ertl, J. Chem. Phys. 100, 1202 (1994).

[14] R. FitzHugh, Biophys. J. 1, 445 (1961).

[15] M. Bär, M. Eiswirth, H. H. Rotermund, and G. Ertl, Phys. Rev. Lett. 69, 945 (1992). 

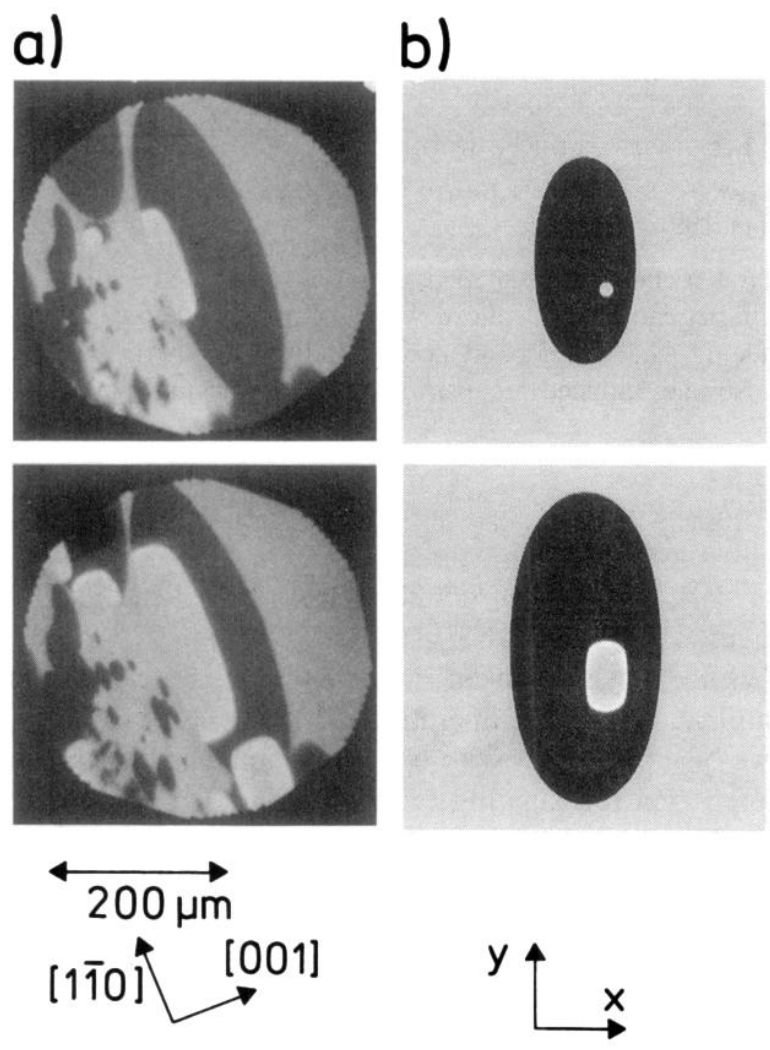

FIG. 1. Simultaneous observation of different front geometries during the $\mathrm{NO}+\mathrm{H}_{2}$ reaction on $\mathrm{Rh}(110)$. (a) Experiment. Two PEEM images recorded with a time interval of 19 s showing subsequent nucleation and growth of oxygen forming (black) and oxygen removing fronts (white). Experimental conditions $T=620 \mathrm{~K}, p_{\mathrm{NO}}=1.8 \times 10^{-6} \mathrm{mbar}$, and $p_{\mathrm{H}_{2}}=5.2 \times 10^{-6}$ mbar. (b) Simulation. Evolution of black and white fronts both from circular initial conditions (the white one was triggered in the first snapshot, 3 time units after the black one). The activator concentration is shown with black denoting higher values. $\left(a=2.1, b=-0.2, \varepsilon=0.025, D_{0}=\right.$ $0.25, D_{1}=1, D_{2}=3$, and $v_{\text {crit }}=0.4415$; explicit integration with $d t=0.001$, grid size $512 \times 512$ corresponding to $70 \times 70$ spatial units). 


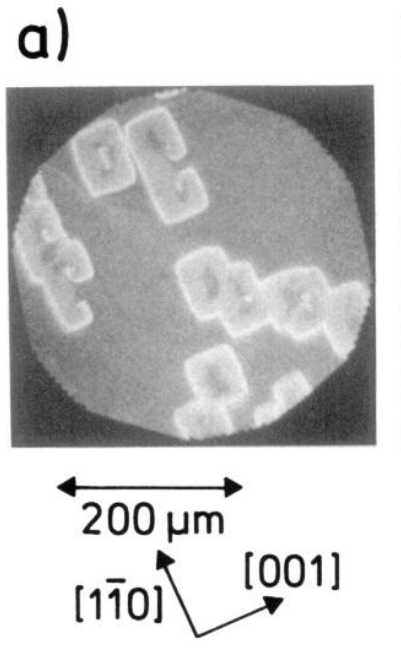

\section{b)}
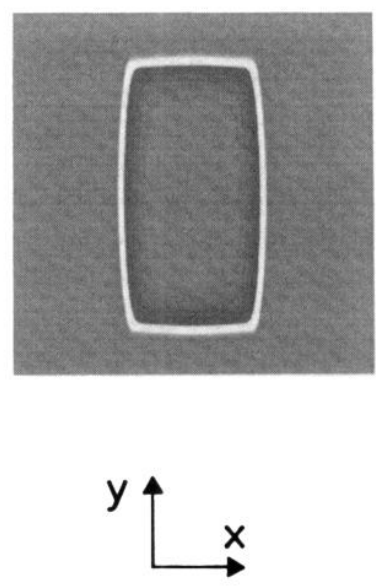

FIG. 3. Rectangularly shaped target pattern in the $\mathrm{NO}+$ $\mathrm{H}_{2}$ reaction on $\mathrm{Rh}(110)$ in the excitable regime. (a) Experiment. PEEM image recorded at $T=620 \mathrm{~K}$ with $p_{\mathrm{NO}}=$ $1.8 \times 10^{-6} \mathrm{mbar}$, and $p_{\mathrm{H}_{2}}=5 \times 10^{-6}$ mbar. (b) Simulation. Snapshot 7 time units after an initial condition of a white circle on the black rest state $[a=0.5, b=-1.04$, and other parameters as in Fig. 1(b). 


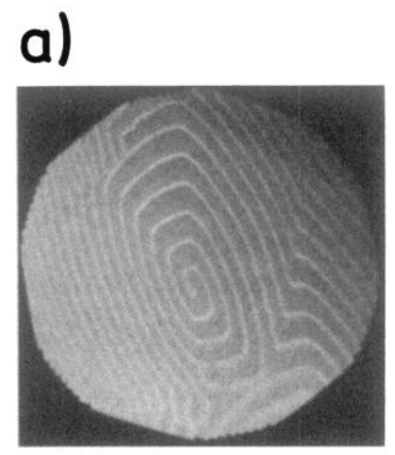

b)
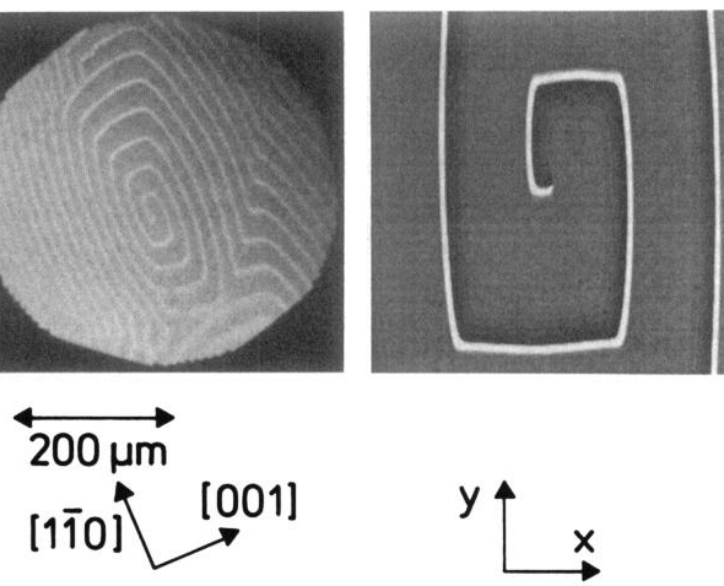

FIG. 4. Rectangularly distorted spiral wave in the NO + $\mathrm{H}_{2}$ reaction on $\mathrm{Rh}(110)$. (a) Experiment. PEEM image recorded at $T=560 \mathrm{~K}$ with $p_{\mathrm{NO}}=1.8 \times 10^{-6} \mathrm{mbar}$ and $p_{\mathrm{H}_{2}}=5.3 \times 10^{-6} \mathrm{mbar}$. The spiral rotates with a frequency of $4.3 \times 10^{-2} \mathrm{~s}^{-1}$. (b) Simulation. Spiral formed after 50 time units from cross-gradient initial conditions [parameters as in Fig. 3(b). 